\title{
Deformation-Based Morphometry and Its Relation to Conventional Volumetry of Brain Lateral Ventricles in MRI
}

\author{
Christian Gaser,* I gor Nenadic,* Bradley R. Buchsbaum,† Erin A. Hazlett, $\ddagger$ and Monte S. Buchsbaum \\ *Department of Psychiatry, Friedrich-Schiller-University, Philosophenweg 3, D-07740 J ena, Germany; ¥Neuroscience PET Laboratory, \\ Department of Psychiatry, Mount Sinai School of Medicine, One Gustave L. Levy Place, New York, New York 10029-6574; and
} †Department of Cognitive Science, University of California, Irvine, California 92697

Received September 5, 2000

Deformation-based morphometry (DBM) is a useful technique to detect morphological differences over the entire brain since it analyses positional differences between every voxel and a standard brain. In this report we compare DBM to semimanual tracing of brain ventricles in a population of 39 patients with schizophrenia. High-resolution $\mathrm{T}_{1}$-weighted magnetic resonance images were obtained and processed with DBM and interactive tracing software. We evaluate the validity of the DBM in two different approaches. First, we divide subjects into two groups based on the mean ventricular/brain ratios and compute statistical maps of displacement vectors and their spatial derivatives. This analysis demonstrates a striking consistency of the DBM and visual tracing results. We show that restricting the information about the deformation fields by computing the local J acobian determinant (as a measure of volume change) provides evidence of the shape of ventricular deformation which is unavailable from ventricular volume measures alone. Second, we compute a mean measure of the J acobian values over the entire ventricles and observe a correlation of $\mathbf{r}=\mathbf{0 . 9 6 2}$ with visual tracing based ventricular/brain ratios. The results support the usefulness and validity of DBM for the local and global examination of brain morphology. 2001 Academic Press

\section{INTRODUCTION}

Morphometric investigations using high-resolution magnetic resonance imaging ( $\mathrm{MRI}$ ) have become an important tool in the study of human brain development and pathology in illnesses such as Alzheimer's disease and schizophrenia. Conventional volumetric methods often use manual or semimanual tracing of brain structures. Although this conventional approach can incorporate detailed anatomical knowledge, it has several drawbacks. Even if tracing measurements are enhanced using semimanual interactive software, obtaining volumetric measures for several brain struc- tures in larger numbers of brains still remains very time-consuming. Hence, most studies rely on relatively small sample sizes and may restrict regions of interest (ROI) to being outlined on only a few MRI slices. In recent years, several automated methods have tried to overcome these problems. One example of a recent alternative approach is deformation-based morphometry (DBM), a tool for detection of structural changes over the entire brain (Bookstein, 1989; Christensen et al., 1997; Grenander and Miller, 1994; Davatzikos et al., 1996; DeQuardo et al., 1996; Haller et al., 1997; I osifescu et al., 1997; Thompson et al., 1997; Ashburner et al., 1998; Gaser et al., 1999; Thirion et al., 2000). However, similar to other voxel-based methods, no direct comparisons to visual tracing vol umetry have been reported.

In this study, we compare DBM to semimanual tracing, the current "gold standard" in volumetric assessment of gross brain morphology. The lateral ventricles were chosen to be assessed by the two methods, since they are among the structures in the brain, which can be delineated best: With their relatively large volume and mostly sharp boundary, tracing the ventricles provides high inter-rater reliabilities. This comparison of the two methods was performed on a population of 39 subjects with schizophrenia. Ventricular enlargement in schizophrenia patients has been demonstrated in many studies (Wright et al., 2000), although not al ways consistently and with considerable variation among subjects. Also, it might be that enlargement occurs preferentially in parts of the ventricles rather than over the entire structure (e.g., Buchsbaum et al., 1997; Sanfilipo et al., 2000). Since many previous studies have measured ventricular volume over the entire left or right lateral ventricle as a whole, a more precise localization of structural differences has not been possible in these approaches.

In this investigation we examine DBM regional sensitivity. A categorical comparison of two subgroups of individuals above and below the mean ventricular/ brain ratio was set up to demonstrate that DBM would 
be able to detect the differences in the lateral ventricles. Within this analysis we use two different levels of information about deformations to test the hypothesis that extracting particular information from the deformation fields yields more local sensitivity to detect volume differences. Given that deformations can result in changes of both volume and position, two of these information levels can be described. First, the displacement vectors of the deformations can be used (as shown in Thompson et al., 1997; Ashburner et al ., 1998; Gaser et al., 1999). Alternatively, the information can be restricted by computing a derivative of the deformation field (Davatzikos et al., 1996). Extracting the local J acobian determinant, a variable common in the physics of continuum mechanics (Gurtin et al., 1987), could help to summarize the information on local volume effects. Finally, we computed a mean measure of the $\mathrm{J}$ acobian values in the region of the ventricles to evaluate how closely this measure correlates with the results of conventional tracing.

\section{METHODS}

\section{Subjects and Scanning Procedure}

Data analysis was performed on MR images of 39 subjects with schizophrenia according to DSM-III R criteria (mean age 37.2 years, SD 12.14; age range 18 to 65 years). Diagnosis was established with the structured CASH interview (Andreasen et al., 1992). Patients with psychiatric or neurological comorbidity (including current substance abuse or dependence) were excluded. High-resolution $T_{1}$-weighted $M R$ images were obtained with a General Electric's Signa 5x system (General Electric's Medical Systems, Milwaukee, $\mathrm{WI})$ using a SPGR pulse sequence $\left(T_{R}=24 \mathrm{~ms}, T_{E}=5\right.$ $\mathrm{ms}$, flip angle $=40^{\circ}$ ) for contiguous 1.2-mm-thick axial slices (in-plane matrix $256 \times 256$, field of view $=23 \mathrm{~cm}$, resulting in a voxel dimension of $0.89 \times 0.89 \times 1.2$ $\mathrm{mm}^{3}$ ). All images were checked for artifacts by visual inspection.

\section{Semimanual Tracing Procedure of the Lateral Ventricles}

Measurements of ventricular volume were performed using the Multi Image Processing Software (MIPS) package (Mount Sinai N euroscience PET Laboratory, New York) as described in an earlier study (Buchsbaum et al., 1997). The ventricles were traced on every axial slice. The image was enhanced with the Sobel gradient filter, producing a usually continuous white line around the ventricular cavity; points were deposited by mouse following an automated local search for the maximum differentiated image intensity value. Typically 30-40 points were placed on a slice and then a spline curve was fitted to the points. The area within the spline curve was then calculated and stored in an ROI file. The lateral ventricle was defined as the portion of the cavity superior to the thalamus and a single contiguous area in each hemisphere. This method, though admittedly time-consuming and somewhat laborious, brings with it the advantage of a constant vigil against unmonitored algorithmic error, and the ability to differentiate the milky encroachment of the choroid plexus (a foil to any automated algorithm) from surrounding tissue- especially on the perimeter of the temporal horns-with similar MRI intensities. The ventricles were outlined by one tracer (BRB) with measured reliability (interclass correlation coefficient of 0.98 on 10 individuals from Buchsbaum et al., 1997). The brain volume was assessed for each person from approximately 20 evenly spaced axial slices following our standard procedure (Hazlett et al., 1999). Ventricular area from every slice was summed to yield total ventricular volume, and this was divided by the whole brain volume to yield the ventricular/brain ratio.

\section{Deformation-Based Morphometry Analysis}

In deformation-based morphometry high-resolution MRI scans of individual subjects are mapped to a template image with three-dimensional nonlinear normalization routines. The basic principle is to analyze these local deformations of an image and to infer local differences in brain structure. This enables the automated measurement of structural alterations over the entire brain (Gaser et al., 1999).

We first resectioned the images to obtain isotropic voxels of $1 \mathrm{~mm}^{3}$. Using a linear normalization (Ashburner et al., 1997), all brains were adjusted for orientation and overall width, length, and height. Thus, the brains are transformed to the anatomical space of a single template brain. This single brain is provided with the SPM99 software package (Wellcome Department of Cognitive Neurology, London) and its space is based on the Talairach space (Talairach and Tournoux, 1988).

Subsequent nonlinear normalization introduced local deformations to each single brain to match it to the same template brain. In comparison to the non-linear normalization algorithm used in a previous study (for details see Gaser et al., 1999) we have now applied the procedure of Kjems et al. (1999), due to it's saving of computation time. The computation time for this nonlinear procedure takes only $330 \mathrm{~s}$ on a $366 \mathrm{MHz}$ Pentium II processor. This approach uses a multigrid algorithm which computes displacement vectors first at a low resolution and uses these results as starting values in the next higher resolution step. Final displacement vectors were obtained from a two times coarser grid than the image resolution of $1 \mathrm{~mm}^{3}$ using a correlation function as voxel similarity measure and the three multi-grid iterations with the parameters of $\alpha=40 / 10$ / 


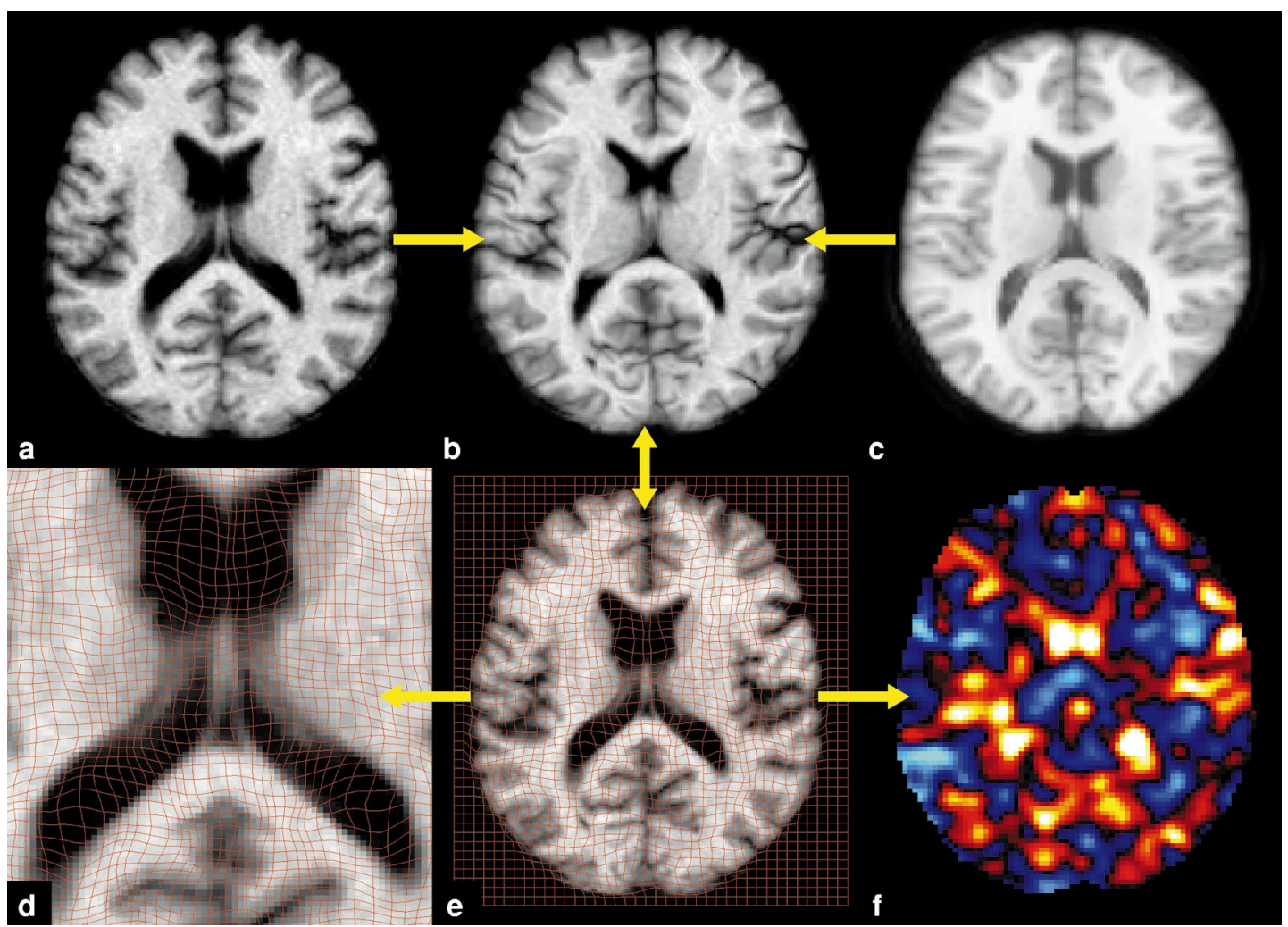

FIG. 1. Steps of analysis in deformation-based morphometry. An example is shown for a single subject in one axial slice. The single object brain (image a) has been corrected for orientation and overall size to the template brain (c). Nonlinear normalization removes most of the anatomical differences between the two brains by introducing local deformations to the object brain, which then (b) looks as similar as possible to the template. Note that these slices only represent two-dimensional information, whereas the method actually works in three dimensions. I mage (e) shows the deformations applied to the object brain by a deformed grid and a magnification of the ventricles is displayed in (d). Analysis for DBM can be either multivariate using these $x-y-z$ displacements of the entire 3-D deformation field or univariate using the local J acobian determinant as a derivative of the field (f).

$5 / 2$ (for the respective resolution steps of computation) and $\beta=2$ (for all steps). Since the normalization routine is based on image intensities, there is no need for surface points or other landmarks.

We obtained three dimensional deformation fields for every brain. Each of these three-dimensional deformation fields consists of displacement vectors for every voxel, which describe the three-dimensional displacement needed to locally deform (warp) the brain to match it to the template (see Fig. 1). The obtained displacement vectors in each voxel were finally smoothed with a Gaussian kernel with FWHM of 8 $\mathrm{mm}$. These images were used in statistical analysis. It is also possible to use derivatives of the deformation fields. The local J acobian determinant is a parameter commonly used in continuum mechanics (Gurtin, 1987), which characterizes volume changes, i.e. local shrinkage or enlargement caused by warping. For this 3-D differential, if all vectors surrounding a pixel are pointing outward from the pixel, a large positive value is generated. If pixels at the current location must be displaced outward, we can infer that the current region at that pixel is smaller than the template and that the regional volume is reduced.

\section{Data Analysis}

Data analysis of DBM results was performed in the overall framework of the general linear model. For the first part of data analysis, we performed a categorical comparison between two subgroups divided on the basis of the mean VBR of 0.1004 of the sample. The first group of 15 subjects (mean age 40.67 years, SD 13.94, age range 18-53 years) had larger ventricles (mean VBR 0.158, SD 0.034), and the second group of 24 patients (mean age 35.12 years, SD 10.67, age range 18-65) had smaller VBR's (mean VBR 0.066, SD $0.015)$. This group comparison included a multivariate and a univariate analysis of variance using a F statistic. The multivariate test (equivalent to the Hotelling's $\mathrm{T}^{2}$ test) looks for differences in the displacement vectors. There are independent groups (large and small ventricles) and three variables-the $x-y-z$ displacements, which characterize the shift needed to locally deform the brain to match it to the template. The 


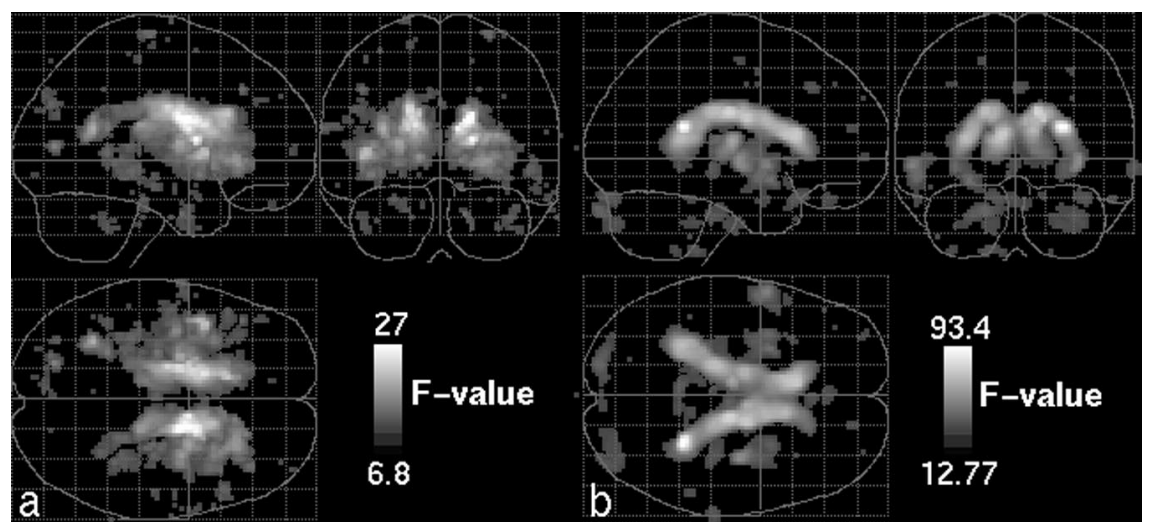

FIG. 2. Maximum intensity projections (MIP) of categorical comparison of subgroups (24 vs 15 patients) using deformation-based morphometry with the multivariate (image a; 3-variate F test with degrees of freedom of 3, 35) and the univariate J acobian approach (image b; univariate $F$ test with degrees of freedom of 1,37 ). In both tests, only voxels with a threshold for significance of $\mathrm{P}<0.001$ are displayed.

univariate approach restricts information of deformations to local volume change only, by using spatial derivatives. We first used the F statistic for both tests, in order to have a direct comparability of statistical assessments. Then we also applied a t test statistic to the univariate design, which allowed differentiation of positive and negative volume changes between both groups. For all statistical tests, a threshold for significance of $\mathrm{P}<0.001$ was applied.
Finally, we compared the results of conventional tracing with an average ventricular measure from DBM. This is comparable to a region analysis. For that purpose we traced the ventricle in the reference brain and defined it as the region of interest (ROI) for all other brains. Then we computed the mean value of the J acobian within this ROI in each deformed brain. This mean measure represents the relative volume change in the ventricles compared to the reference brain. The

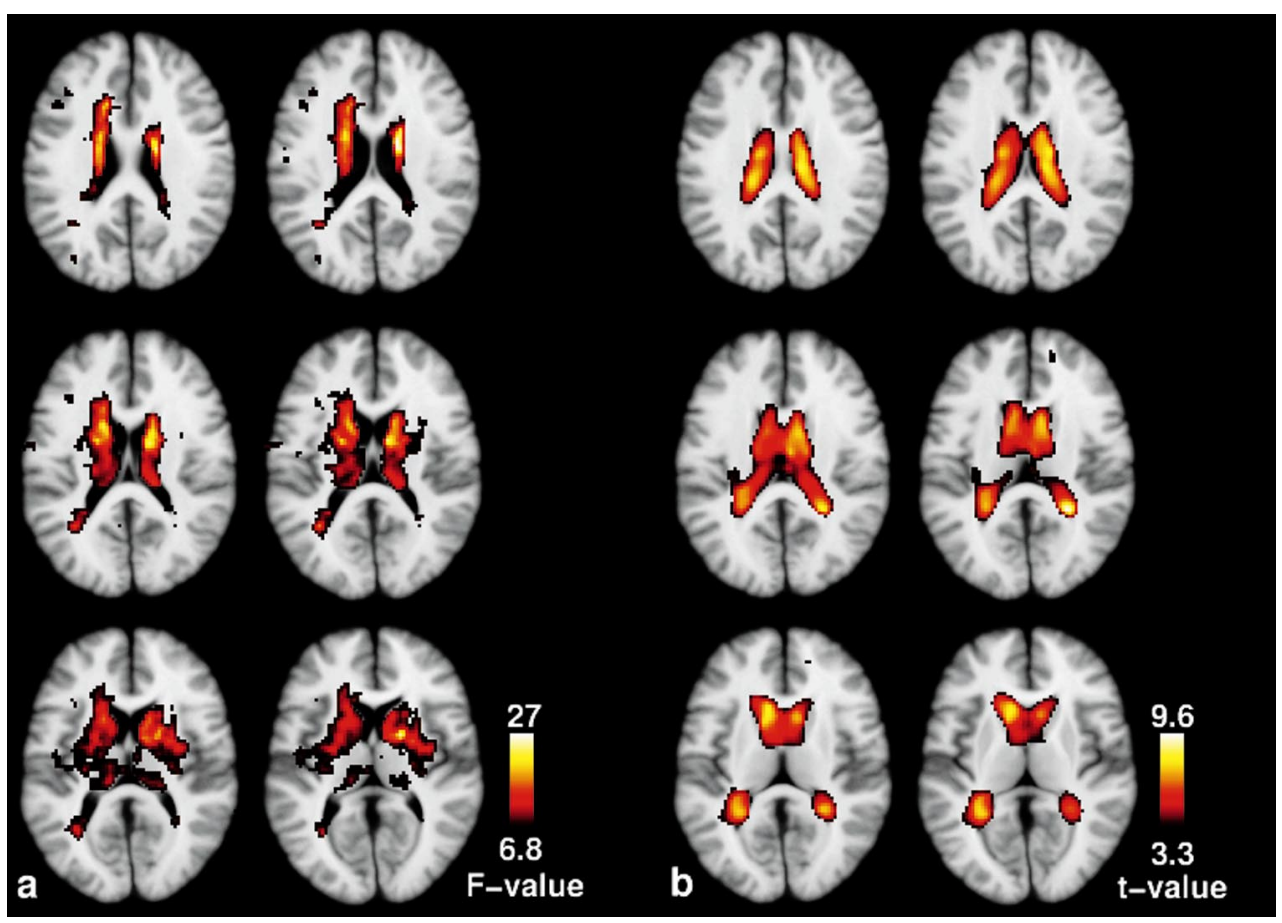

FIG. 3. A few representive transverse sections of the categorical comparison of 24 schizophrenia patients with larger ventricles vs 15 with smaller ventricles using deformation-based morphometry are shown with a multivariate $F$ test (a) and a univariate t test (b). The statistical results were thresholded at $P<0.001$ for each voxel and superimposed on the average image of the 39 nonlinearly normalized brains. The distance between the consecutive slices is $3 \mathrm{~mm}$. 
correlation between this mean J acobian value and the ventricular/brain ratio measures of the conventional tracing was calculated.

\section{RESULTS}

The comparison between 15 patients with large ventricles and 24 patients with small ventricles showed a large cluster of voxels spanning almost the entire lateral ventricle area. In Fig. 2 this comparison is performed with a $F$ test for multivariate (analysis of $x-y-z$ displacements, Fig. 2a) and univariate (analysis of J acobian determinant, Fig. 2b). Results are displayed as maximum intensity projections, which show highest values within each orientation. Note that use of the J acobian determinant (Fig. 2b) shows a clearer delineation of the ventricular system. Figure 3 shows the multivariate and univariate statistical results in selected transverse sections. Since for the univariate test we were only interested in areas with volume increase in the group with large ventricles, this contrast was assessed with a 1-tailed t test statistics. Again, for both methods (Figs. 3a and $3 \mathrm{~b}$ ) the ventricular enlargement was detected. These sections demonstrate that the clusters in the multivariate analysis extend over the ventricular borders (for example into the frontal white matter rostral to the superior lateral portion and into the insular region). With the local J acobian measure, the volume increase changes appear almost exclusively in the ventricle and its borders.

The correlation between the ventricular/brain ratio of conventional visual tracing and the mean J acobian measure in the ventricle as region of interest is $r=$ 0.962 ( $P<0.001)$ is shown in Fig. 4.

\section{DISCUSSION}

In this report, we showed that deformation-based morphometry is able to assess ventricular enlargement, in a way similar to that obtained with conventional volumetric tracing of the structure. The study demonstrated the regional sensitivity of DBM and advantages due to automated assessment.

The results of the categorical comparison have demonstrated two main findings. First, it confirms our suggestion that multivariate statistical images of displacements while providing more information and perhaps being more sensitive to group differences, may be more difficult to interpret since they combine position displacement as well as local size difference indications. In contrast, the univariate images of the J acobian determinant, while not providing information on the directional displacement of structures, may be easier to interpret since they reveal regional structural size alterations which in turn may relate to current biological theories. Although clusters in Figs. 2a and 2b (and $3 a$ and $3 b$, respectively) are roughly similar, the latter

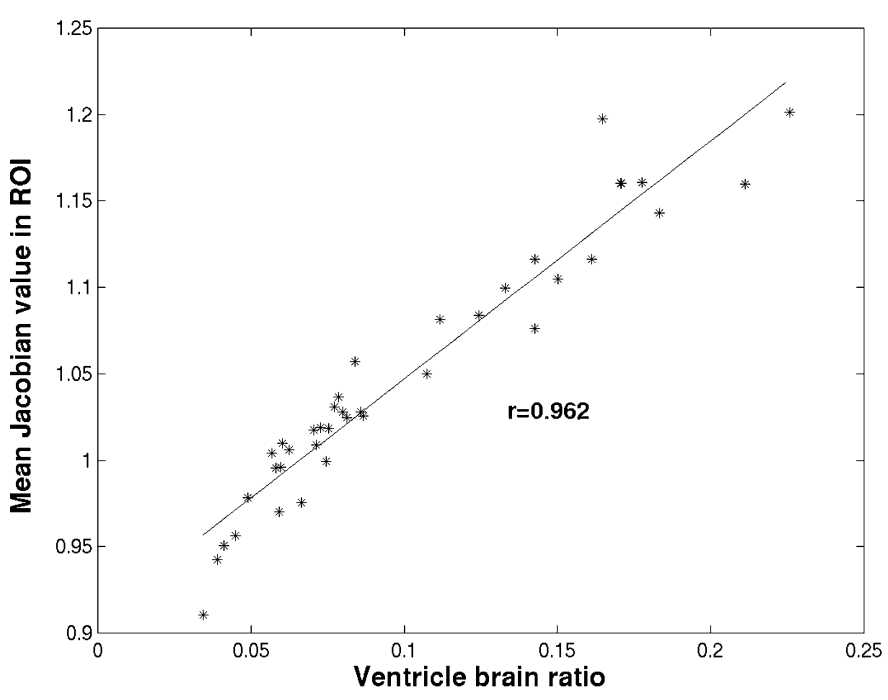

FIG. 4. Correlation between the results of conventional tracing and deformation-based morphometry (DBM). The diagram shows the relation between the ventricular/brain ratio measures of conventional tracing and the mean J acobian value in the ventricles as region of interest (ROI). The ROI was defined in the reference brain and the J acobian values for each subject embodied in this ROI were averaged to get a global measure of the DBM results in this anatomical region.

shows more compact and locally confined structure and clear shape of ventricular boundaries. This is even more obvious by only focusing on the volume increases as shown in Fig. 3b. Using the information of the displacement vectors might prove useful in applications when both location and volume shifts are to be detected. Focusing on volumetric changes, however, the local J acobian is a superior alternative. Another advantage of the local J acobian determinant is apparent when considering that test statistics can directly test whether local shrinkage or enlargement occurs. In multivariate designs, post hoc testing might be necessary to clarify whether changes are due to volume increase or decrease.

Second, these analyses underline the advantages of DBM which operates on a voxel level. This is particularly seen when examining the location of significant pixel clusters of change. Figure 3 indicates that ventricular enlargement appears to affect the lateral surface of the lateral ventricle and the anterior horn and extends out into the region of the insula and back along the anterior surface of the posterior inferior portion of the corpus callosum; indeed the callosum seems to be restraining anatomical deformation in this region. It should be clearly noted that these effects are related to the type of ventricular enlargement seen in schizophrenics with large ventricles in comparison to those with small ventricles, and do not necessarily reflect shape change seen in normal ventricles with age, hydrocephalus, Alzheimer's disease, or other brain atrophy or ventricular enlargement conditions. However, 
now that validity of the DBM and J acobian measures has been demonstrated, we believe that its application to these pathophysiological conditions will be useful.

The strong correlation between the average measure of the Jacobian within a predefined region and the ventricular/brain ratio measure provides an extension of this validation issue to structure volume measurement. Mean measures of the DBM within one region are strongly related to results of conventional tracing of this region (Fig. 4). This indicates that volume changes within a predefined region can be obtained by tracing this area only once in the reference brain.

In conclusion, this analysis of ventricular volume differences suggests the usefulness and validity of DBM methodology for morphometric analyses. Fully automated image processing and three-dimensional assessment over the entire brain gives several advantages over volumetric measures by tracing, even when a region of interest such as the ventricles can be easily delineated. Processing of the deformation fields, as performed by computation of the local J acobian determinant might be necessary for enhancement of selective aspects of differences in brain morphology. Our results might apply to other morphometry tools which are based on warping techniques. Both intensity and surface-based methods generate 3-D deformation fiel ds on which J acobian measure of volume changes can be calculated and statistically analyzed.

With this demonstration of validity, deformationbased morphometry emerges as a highly useful tool for the examination of brain morphology from MRI, allowing an unbiased automated analysis of the whole brain with high regional sensitivity.

\section{ACKNOWLEDGMENTS}

The authors thank Dr. M. Mehmet Haznedar, Dr. Lina Shihabuddin, and coworkers at Mount Sinai Medical Center, New York, for subject referral and clinical interviews. Parts of this work were supported by NIMH Grants MH40071 and MH56489, a grant from the Dana Foundation to Dr. Buchsbaum, the National Alliance for Research on Schizophrenia and Depression (NARSAD), and the VKF Clinical Research Council of the University of J ena.

\section{REFERENCES}

Bookstein, F. L. 1989. Principal warps: Thin-plate splines and the decomposition of deformations. IEEE Trans. Patt. Anal. Machine Intell. 11: 567-585.

Andreasen, N. C., Flaum, M., and Arndt, S. 1992. Comprehensive assessment of symptoms and history (CASH): An instrument for assessing diagnosis and psychopathology. Arch. Gen. Psychiatry 49: 615- 623.

Ashburner, J ., Neelin, P., Collins, D. L., Evans, A., and Friston, K. 1997. Incorporating prior knowledge into image registration. Neurol mage 6: 344-352.
Ashburner, J ., Hutton, C., Frackowiak, R., J ohnsrude, I., Price, C., and Friston, K. 1998. Identifying global anatomical differences: Deformation-based morphometry. Hum. Brain Mapp. 6: 348-357.

Buchsbaum, M. S., Yang, S., Hazlett, E., Siegel, B. V. J r., Germans, M., Haznedar, M., O'Flaithbheartaigh, S., Wei, T., Silverman. J ., and Siever, L. J. 1997. Ventricular volume and asymmetry in schizotypal personality disorder and schizophrenia assessed with magnetic resonance imaging. Schizophren. Res. 27: 45-53.

Christensen, G. E., J oshi, S. C., and Miller, M. I. 1997. Volumetric transformation of brain anatomy. IEEE Trans. Med. Imag. 16: 864- 877.

Davatzikos, C., Vaillant, M., Resnick, S. M., Prince, J . L., Letovsky, S., and Bryan, R. N. 1996. A computerized approach for morphological analysis of the corpus callosum. J . Comput. Assist. Tomogr. 20: 88-97.

DeQuardo, J . R., Bookstein, F. L., Green, W. D., Brunberg, J . A., and Tandon, R. 1996. Spatial relationships of neuroanatomic landmarks in schizophrenia. Psychiatry Res. 67: 81-95.

Gaser, C., Volz, H.-P., Kiebel, S., Riehemann, S., and Sauer, H. 1999. Detecting structural changes in whole brain based on nonlinear deformations-Application to schizophrenia research. Neuroimage 10: 107-113.

Grenander, U., and Miller, M. I. 1994. Representations of knowledge in complex systems. J . R. Statist. Soc. B 56: 549- 603.

Gurtin, M. E. 1987. An Introduction to Continuum Mechanics. Academic Press, Boston.

Hazlett, E. A., Buchsbaum, M. S., Byne, W., Wie, T. C., SpiegelCohen, J., Geneve, C., Kinderlehrer, R., Haznedar, M. M., Shihabuddin, L., and Siever, L. J . 1999. Three dimensional analysis with MRI and PET of the size, shape, and function of the thalamus in the schizophrenia spectrum. Am. J. Psychiatry 156: 1190-1199.

Haller, J . W., Banerjee, A., Christensen, G. E., Gado, M., J oshi, S., Miller, M. I., Sheline, Y., Vannier, M. W., and Csernansky, J . G. 1997. Three-dimensional hippocampal MR morphometry with high-dimensional transformation of a neuroanatomic atlas. Radiology 202: 504-510.

Kjems, U., Strother, S. C., Anderson, J ., Law, I., and Hansen, L. K. 1999. Enhancing the multivariate signal of $\left[{ }^{15} \mathrm{O}\right]$ water PET studies with a new nonlinear neuroanatomical registration algorithm. IEEE Trans. Med. Imag. 18: 306-319.

I osifescu, D. V., Shenton, M. E., Warfield, S. K., Kikinis, R., Dengler, J ., J olesz, F. A., and McCarley, R. W. 1997. An automated registration algorithm for measuring MRI subcortical brain structures. Neurol mage 5: 13-25.

Sanfilipo, M., Lafargue, T., Arena, L., Rusinek, H., Kushner, K., Lautin, A., Loneragan, C., Vaid, G., Rotrosen, J ., and Wolkin, A. 2000. Fine volumetric analysis of the cerebral ventricular system in schizophrenia: Further evidence for multifocal mild to moderate enlargement. Schizophren. Bull 26: 201-216.

Talairach, J ., and Tournoux, P. 1988. Co-Planar Stereotaxic Atlas of the Human Brain. Thieme, New York.

Thirion, J .-P., Prima, S., Subsol, S., and Roberts, N. 2000. Statistical analysis of normal and abnormal dissymmetry in volumetric medical images. Med. Image Anal. 4: 111-121.

Thompson, P. M., MacDonald, D., Mega, M. S., Holmes, C. J ., Evans, A. C., and Toga, A. W. 1997. Detection and mapping of abnormal brain structure with a probabilistic atlas of cortical surfaces. J . Comp. Assist. Tomogr. 21: 567-581.

Wright, I. C., Rabe-Hesketh, S., Woodruff, P. W. R., David, A. S., Murray, R. M., and Bullmore, E. T. 2000. Meta-analysis of regional brain volumes in schizophrenia. Am. J. Psychiatry 157: 16-25. 